\title{
The Isomeric Composition of Hydroperoxides Formed by Autoxidation of Unsaturated Triglycerides and Vegetable Oils
}

\author{
Dong Ki Park, Junji Terao and Setsuro Matsushita \\ Research Institute for Food Science, Kyoto University, \\ Kyoto 611, Japan \\ Received February 27, 1981
}

\begin{abstract}
Trioleoylglycerol (TO), trilinoleoylglycerol (TL), and trilinolenoylglycerol (TLN)were autoxidized in the dark at $37^{\circ} \mathrm{C}$. Monohydroperoxides (MHP), the primary products, were isolated by preparative thin-layer chromatography (TLC). The isomeric compositions of their hydroperoxy fatty acid components were determined by gas chromatography-mass spectrometry (GC-MS) as follows-TO: the 8-, 9-, 10-, and 11-isomers; TL: the 9-, and 13-isomers; and TLN: the 9-, 12-, 13-, and 16-isomers. The proportions of isomers in each MHP did not vary with the oxidation time. The isomeric compositions of hydroperoxy fatty acid components obtained from autoxidized soybean and olive oils indicated that each unsaturated fatty acyl group of triacylglycerol ( $T G$ ) in vegetable oils produced isomeric hydroperoxides during autoxidation in a way similar to the corresponding fatty acid methyl esters. The proportions of the isomers obtained from autoxidized oils changed with the level of oxidation. Isomers coming from linolenic acid in soybean oil and those from linoleic acid in olive oil decreased remarkably at a high level of oxidation.
\end{abstract}

It is well known that autocatalytic autoxidation of edible oils proceeds by the mechanism of a free radical chain reaction resulting in the accumulation of hydroperoxides and a variety of secondary oxidation products. Earlier works have demonstrated that isomeric hydroperoxides are formed by autoxidation of unsaturated fatty acid. ${ }^{1)}$ The isomeric compositions of hydroperoxides formed by autoxidation of methyl oleate, methyl linoleate, and methyl linolenate have already been investigated in detail by high performance liquid chromatography (HPLC), ${ }^{2 \sim 4)}$ and gas chromatography mass spectrometry (GC-MS). ${ }^{5}$ 8) Recently, Frankel et al., ${ }^{9)}$ characterized the isomeric hydroperoxides of autoxidized fatty acid methyl ester mixture prepared from soybean oil, and estimated the contribution of each unsaturated fatty acid methyl ester to the isomeric distribution of hydroperoxide isomers. However, only a few investigations ${ }^{10,11)}$ have been carried out to characterize the isomeric hydroperoxides of unsaturated triacylglycerol (TG). Furthermore, no studies have been reported on the isomeric composition of hydroperoxides obtained from autoxidized vegetable oils, although vegetable oils consist of several molecular species of TG.

In the preceding papers, ${ }^{12,13)}$ we isolated hydroperoxides from photosensitized oxidation products of unsaturated TG, and succeeded in determinating the isomeric distribution of hydroperoxy fatty acid components by using hydrogenation, methanolysis, and GC-MS analysis. The purpose of this work is to clarify the structure of isomeric hydroperoxides formed by autoxidation of vegetable oils. Trioleoylglycerol (TO), trilinoleoylglycerol (TL), trilinolenolyglycerol (TLN), soybean oil and olive oil were selected and were subjected to autoxidation. The isomeric compositions of hydroperoxy fatty acid components produced during autoxidation were determined by the same procedure as described in the preceding paper. ${ }^{13)}$ Analysis of the isomeric distribution of hydroperoxides will give useful information for vegetable oil autoxidation. 


\section{MATERIALS AND METHODS}

Materials. TO, TL, and TLN, all $99 \%$ grade, were purchased from Serdary Research Laboratories, Inc., London. These unsaturated TG were purified by column chromatography using Florisil (100 200 mesh) to get rid of any peroxides. Soybean oil and olive oil were obtained from Nakarai Chem., Ltd., Kyoto. Soybean oil TG was prepared from unoxidized soybean oil by treatment with active charcoal and Florisil column chromatography with eluting hexane.

Autoxidation. Oil $(1.0 \sim 5.0 \mathrm{~g})$ in a glass vial was autoxidized by incubating at $37^{\circ} \mathrm{C}$ in the dark. The peroxide value was measured according to the method of Asakawa and Matsushita. ${ }^{\text {(4) }}$

Isolation of hydroperoxides. Autoxidized TG (100 mg) was applied to a TLC plate ( $1.25 \mathrm{~mm}$ thick) coated with silica gel (Merck, silika gel PF-254) in a solvent for development, hexane and ethyl ether $(8: 7)$. The bands of the hydroperoxides were detected under ultraviolet light and were scraped off, and then extracted with chloroform three times. IR spectra and ${ }^{1} \mathrm{H}-\mathrm{NMR}$ spectra were taken with a Hitachi Model 285 spectrometer and a HitachiPerkin Elmer Model $90(90 \mathrm{MHz})$, respectively.

Determination of the isomeric compositions of hydroperoxy fatty acid components. The isomeric compositions of the hydroperoxy fatty acid components of oxidized oil or TG hydroperoxides were determined by hydrogenation, methanolysis, and mass chromatographic analysis of trimethylsilyl derivatives of resulting hydroxy fatty acid methyl esters by procedure as described in the preceding paper. ${ }^{13)}$

\section{RESULTS AND DISCUSSION}

Figure 1 shows the change in peroxide value during autoxidation. The stability in resisting autoxidation apparently increased in the following order: TLN $<$ TL $<$ soybean oil TG $<$ soybean oil $<$ olive oil $<$ TO. The time taken to reach $100 \mathrm{meq} / \mathrm{kg}$ of peroxide value was estimated as follows: TO : 72 days; TL : 2 days; TLN : 0.4 day; soybean oil TG: 6 days; soybean oil : 20 days; olive oil : 48 days. Earlier work $^{15)}$ found that the autoxidation rates of methyl oleate, methyl linoleate and methyl linolenate were $1: 12: 25$. A similar relationship was observed in the stability among the corresponding TG, that is, TO, TL and TLN, although TO appeared to be highly resistant to oxidation as compared with TL and TLN. It

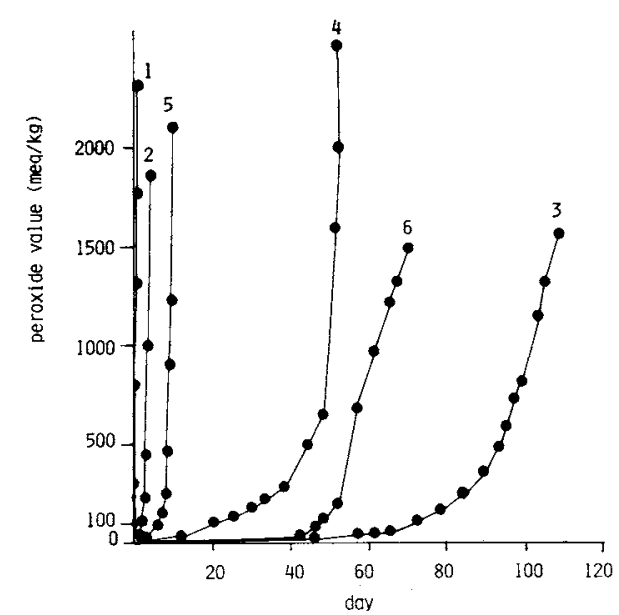

FIG. 1. The Change of Peroxide Value with Oxidation Time.

Each oil and TG $(70 \mathrm{mg})$ in a glass vial (I.D., $1.2 \mathrm{~cm}$ ) was autoxidized by incubating in the dark at $37^{\circ} \mathrm{C} .1, \mathrm{TLN} ; 2$, TL; 3, TO; 4, soybean oil; 5 , soybean oil TG; 6 , olive oil.

seems likely that the stability of vegetable oils to oxidation is affected significantly by their fatty acid compositions, tocopherol contents, and peroxide contents represented by peroxide value. Prior to the autoxidation experiment, peroxide values of unsaturated TO. TL, and soybean oil TG were lower than $1.0 \mathrm{meq} / \mathrm{kg}$, and those of soybean oil and olive oil were 3 and $12 \mathrm{meq} / \mathrm{kg}$, respectively. The tocopherol content of soybean oil is considered to be larger than that of olive oil. ${ }^{16)}$ The fatty acid compositions of the two oils were determined as follows:

soybean oil : palmitic acid $(16: 0)=9 \%$; stearic acid $(18: 0)=4 \%$; oleic acid $(18: 1)=23 \%$; linoleic acid $(18: 2)=56 \%$; linolenic acid $(18: 3)=8 \%$.

olive oil: $16: 0=12 \% ; 18: 0=3 \% ; 18: 1=$ $79 \% ; 18: 2=6 \%$.

The results indicating that soybean oil was less stable to autoxidation than olive oil (Fig. 1) can be explained by the difference of the fatty acid compositions, especially $18: 2$ and $18: 3$. It is apparent that soybean oil TG is more susceptible to oxidation than soybean oil because of the lack of tocopherol in soybean oil TG.

Figure 2 shows the typical pattern of oxi- 
dized TL on TLC. Oxidation products, A and $B$, were isolated from the unoxidized TL by preparartive TLC. IR spectra of both compounds gave strong absorption at $3,450 \mathrm{~cm}^{-1}$

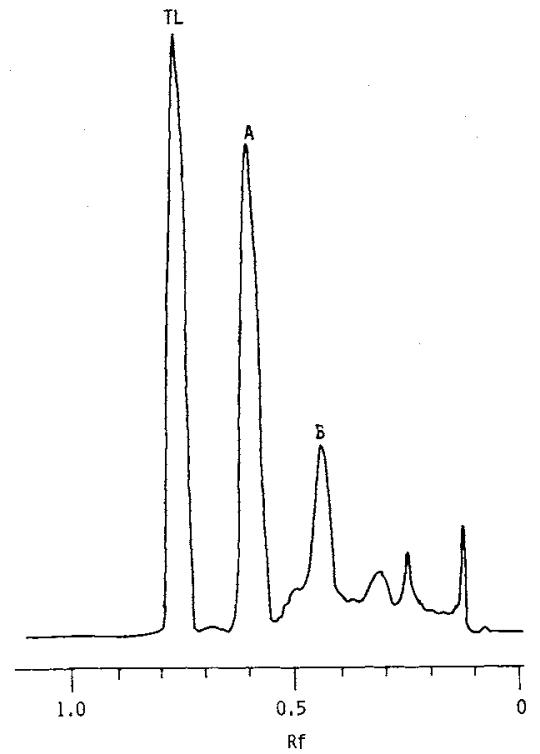

Frg. 2. The Densitometric Pattern of Oxidized TL on a TLC Plate.

An aliquot of the oxidized TL (peroxide value $=1,400$ $\mathrm{meq} / \mathrm{kg}$ ) was spotted on the TLC plate (Merck, silica gel PE-254, $0.25 \mathrm{~mm}$ thick). The plate was developed with a solvent of hexane and ethyl ether $(8: 7)$. After it was sprayed with $50 \% \mathrm{H}_{2} \mathrm{SO}_{4}$ in a saturated $\mathrm{K}_{2} \mathrm{CrO}_{7}$ solution, the plate was heated for $10 \mathrm{~min}$, prior to monitoring by a densitometer at $350 \mathrm{~nm}$. attributed to the hydroperoxy group. ${ }^{17)}$ The IR spectra and peroxide values of 2,500 (A) and 4,300 meq/ $\mathrm{kg}$ (B) well agreed with those obtained from monohydroperoxides (MHP) and dihydroperoxides (DHP) of autoxidized safflower oil. ${ }^{10,11)}$ Furthermore, NMR spectra of A and B show broad signals at downfield, $c a .7 .7 \mathrm{ppm}$, as a result of hydroperoxy protons. The number of hydroperoxy protons calculated from the integral based on the terminal methyl protons were 1.1 (A) and 2.1 (B), respectively. UV spectra of both compounds gave the same absorption maxima at $233 \mathrm{~nm}$ in hexane (A, $\varepsilon=21,000 ; \mathrm{B}, \varepsilon=43,000)$ due to cojugated diene. Thus, compound $A$ was identified as TL-MHP, consisting of two linoleoyl groups and one hydroperoxylinoleoyl group; and compound B was identified at TL-DHP, consisting of one linoleoyl group and two hydroperoxylinoleoyl groups. Oxidized TO and TLN also gave a similar TLC pattern, as shown in Fig. 2, and MGP of TO and TLN were prepared by the same procedure at TL-MHP.

Table I shows the isomeric compositions of hydroperoxy fatty acid components obtained from MHP of TO, TL, and TLN. TO yielded almost equal amounts of the 8-, 9-, 10-, and 11-isomers, and TL yielded almost equal amounts of the 9- and 13-isomers. Four iso-

Table I. The Isomeric Compositions of Methyl Hydroxy OCtadecanoate OBTAINED FROM MHP OF UNSATURATED TG

\begin{tabular}{|c|c|c|c|c|c|c|c|c|c|c|}
\hline \multirow{2}{*}{ TG } & \multirow{2}{*}{$\begin{array}{c}\text { Peroxide } \\
\text { value }(\mathrm{meq} / \mathrm{kg})\end{array}$} & \multicolumn{9}{|c|}{ Percent composition } \\
\hline & & 8-OH & 9-OH & $10-\mathrm{OH}$ & $11-\mathrm{OH}$ & $12-\mathrm{OH}$ & $13-\mathrm{OH}$ & $14-\mathrm{OH}$ & $15-\mathrm{OH}$ & $16-\mathrm{OH}$ \\
\hline \multirow{3}{*}{ TO } & 670 & 27 & 24 & 20 & 29 & - & - & - & - & - \\
\hline & 1300 & 26 & 22 & 22 & 30 & - & - & - & - & 一 \\
\hline & 1600 & 25 & 22 & 22 & 31 & $\cdots$ & - & - & - & - \\
\hline \multirow{4}{*}{ TL } & 51 & - & 49 & - & - & - & 51 & - & - & - \\
\hline & 170 & $\ldots$ & 48 & - & - & - & 52 & - & - & - \\
\hline & 230 & _- & 53 & - & - & - & 47 & - & - & - \\
\hline & 2500 & - & 53 & - & - & - & 47 & - & - & - \\
\hline \multirow{4}{*}{ TLN } & 89 & - & 37 & - & - & 6 & 11 & - & - & 46 \\
\hline & 390 & - & 39 & - & - & 7 & 8 & - & - & 46 \\
\hline & 540 & - & 38 & - & - & 7 & 8 & - & - & 47 \\
\hline & 940 & - & 39 & - & - & 8 & 9 & & & 44 \\
\hline
\end{tabular}


TABle II. The Isomeric Compositions of Methyl Hydroxy Octadecanoate Obtained from Autoxidized Soybean Oil and Soybean Oil TG

\begin{tabular}{|c|c|c|c|c|c|c|c|c|c|}
\hline \multirow{2}{*}{$\begin{array}{c}\text { Peroxide } \\
\text { value }(\mathrm{meq} / \mathrm{kg})\end{array}$} & \multicolumn{9}{|c|}{ Percent composition } \\
\hline & $8-\mathrm{OH}$ & 9-OH & $10-\mathrm{OH}$ & $11-\mathrm{OH}$ & $12-\mathrm{OH}$ & $13-\mathrm{OH}$ & 14-OH & $15-\mathrm{OH}$ & $16-\mathrm{OH}$ \\
\hline \multicolumn{10}{|l|}{ Soybean oil } \\
\hline 19 & 2 & 39 & (5) & $\operatorname{tr}$. & 10 & 39 & - & - & 5 \\
\hline 64 & 1 & 44 & 2 & $\operatorname{tr}$ & 6 & 41 & - & - & 6 \\
\hline 93 & 1 & 46 & 2 & tr. & 4 & 40 & - & - & 7 \\
\hline 110 & 1 & 47 & 2 & tr. & 3 & 41 & - & $\ldots$ & 6 \\
\hline 210 & 1 & 46 & 3 & tr. & 3 & 41 & $\ldots$ & - & 4 \\
\hline $470^{a}$ & 3 & 45 & 3 & 1 & 3 & 41 & - & - & 4 \\
\hline $1400^{a}$ & 2 & 47 & 2 & 2 & tr. & 46 & - & - & 1 \\
\hline \multicolumn{10}{|l|}{ Soybean oil TG } \\
\hline 26 & 5 & 45 & 3 & 3 & (3) & 37 & & - & (4) \\
\hline 58 & 4 & 46 & 3 & 3 & (3) & 39 & - & - & (3) \\
\hline 140 & 3 & 49 & 2 & 2 & tr. & 41 & - & - & 3 \\
\hline $480^{a}$ & 3 & 48 & 1 & 2 & tr. & 44 & - & - & (2) \\
\hline
\end{tabular}

tr.: trace amounts (less than $1 \%$ ).

Percent in parentheses: $\alpha$-cleavage ions of the isomers were detected incompletely in the mass chromatogram.

a MHP fractions of oxidized oil were used for the determination of isomeric composition.

mers, the 9-, 12-, 13- and 16-isomers, were obtained from MHP of TLN, in which the proportions of the 9- and 16-isomers were apparently higher than those of the 12- and 13-isomers. The isomeric distribution obtained from TL-DHP was almost the same as that of the TL-MHP (data are not shown here). Quantitative ratios of the isomers in each TG did not change with the peroxide value of oxidized TG, and were in accordance with those of the corresponding fatty acid methyl ester reported by Frankel et al. ${ }^{6 / 8)}$ It is therefore concluded that each unsaturated fatty acyl group of $\mathrm{TG}$ is oxidized in a manner similar to fatty acid methyl esters to produce isomeric hydroperoxides.

Isomeric distributions of hydroperoxy fatty acid components of oxidized soybean oil and soybean oil TG were determined as shown in Table II. Hydroperoxy fatty acid components consisted of the 9- and 13-isomers predominantly, although small amounts of 8-, 11-, 12-, and 16-isomers were also present in the composition. From the results shown in Table $\mathrm{I}$, the isomeric hydroperoxy fatty acid components were formed by autoxidation of each unsaturated fatty acyl group as follows: the 8-,

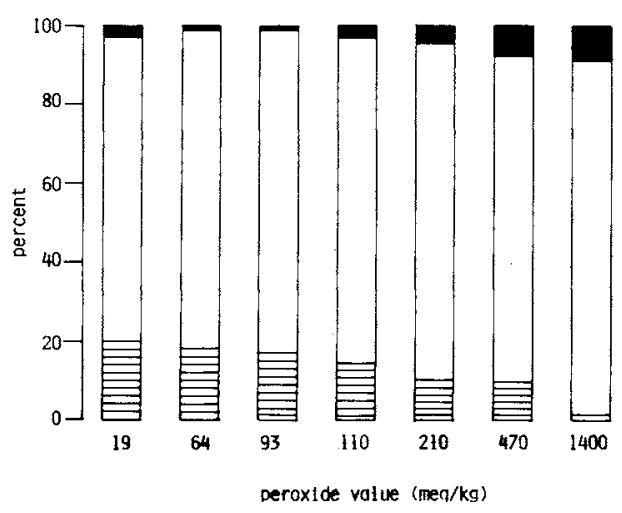

FIG. 3. The Proportion of Unsaturated Fatty Acyl Group of Soybean Oil to the Formation of Hydroperoxy Fatty acid Components.

口, 18:1; $\square, 18: 2 ;$ 冒 $, 18: 3$.

The proportion of each acyl group was calculated as follows:

$18: 3=(12-\mathrm{OH})+(16-\mathrm{OH})+0.78 \times(16-\mathrm{OH})$

$+0.19 \times(16-\mathrm{OH})$

$18: 2=1.0 \times[(13-\mathrm{OH})-0.19 \times(16-\mathrm{OH})]$

$+[(13-\mathrm{OH})-0.19 \times(16-\mathrm{OH})]$

$18: 1=100-(18: 3+18: 2)$

$0.78 \times(16-\mathrm{OH}): 9-\mathrm{OH}$ from $18: 3$,

$0.19 \times(16-\mathrm{OH}): 13-\mathrm{OH}$ from 18.3 ,

$1.0 \times[(13-\mathrm{OH})-0.19 \times(16-\mathrm{OH})]: 9-\mathrm{OH}$ from $18: 2$,

$(13-\mathrm{OH})-0.19 \times(16-\mathrm{OH}): 13-\mathrm{OH}$ from $18: 2$. 
Table III. The Isomeric Compositions of Methyl Hydroxy Octadecanoate Obtained from Autoxidized Olive Oil

\begin{tabular}{cccccccccc}
\hline \multirow{2}{*}{$\begin{array}{c}\text { Peroxide } \\
\text { value (meq/kg) }\end{array}$} & \multicolumn{8}{c}{ Percent composition } \\
\cline { 2 - 9 } & $8-\mathrm{OH}$ & $9-\mathrm{OH}$ & $10-\mathrm{OH}$ & $11-\mathrm{OH}$ & $12-\mathrm{OH}$ & $13-\mathrm{OH}$ & $14-\mathrm{OH}$ & $15-\mathrm{OH}$ & $16-\mathrm{OH}$ \\
\hline 30 & 4 & 45 & 15 & 6 & - & 30 & - & - & - \\
130 & 16 & 32 & 14 & 18 & - & 20 & - & - & - \\
490 & 19 & 29 & 15 & 20 & - & 17 & - & - & - \\
960 & 24 & 27 & 20 & 24 & - & 5 & - & - & - \\
$2500^{a}$ & 22 & 24 & 22 & 32 & - & - & - & - & - \\
\hline
\end{tabular}

a MHP fractions of oxidized oil were used for the determination of isomeric composition.

10-, and 11-isomers come from 18:1 exclusively; the 9-isomer, from 18:1, 18:2 and $18: 3$; the 13 -isomer, from $18: 2$ and $18: 3$; the 12- and 16-isomers, from 18:3 exclusively. Quantitative ratios of the isomers changed with the oxidation level. In particular, 12- and 16-isomers coming from $18: 3$ decreased at a higher oxidation level (peroxide value $=1,400$ $\mathrm{meq} / \mathrm{kg}$ ). The 8-, 10-, and 11-isomers derived from 18:1 were detected from the low level of oxidation (peroxide value $=19 \mathrm{meq} / \mathrm{kg}$ ). The contribution of each unsaturated fatty acyl group to the formation of isomeric hydroperoxides was calculated according to the results in Table I, and was shown in Fig. 3. The isomeric distributions of each fatty acyl group, calculated according to the scheme shown in the legend of Fig. 3, well agreed with those obtained from fatty acid methyl esters ${ }^{(\sigma)}$ and unsaturated TG shown in Table $I$ at a higher level of oxidation. Hydroperoxy fatty acid components from 18:3 decreased, and those from 18:1 increased with the oxidation level. However, at a lower oxidation level (peroxide value $=19 \mathrm{meq} / \mathrm{kg}$ ), the proportion of the 12 isomer $(10 \%)$ was significantly higher than was expected to come from 18:3. Frankel et al. ${ }^{9,18)}$ also found the unusual proportion of the 12-isomer in oxidized soybean oil methyl esters, and suggested that this isomer came from photosensitized oxidation of $18: 2$. In the preceding paper, ${ }^{13}$ ) we demonstrated that photooxidation of soybean oil yielded considerable amounts of the 10-, and 12-isomers due to the occurrence of photosensitized oxidation of $18: 2$. Photosensitized oxidation

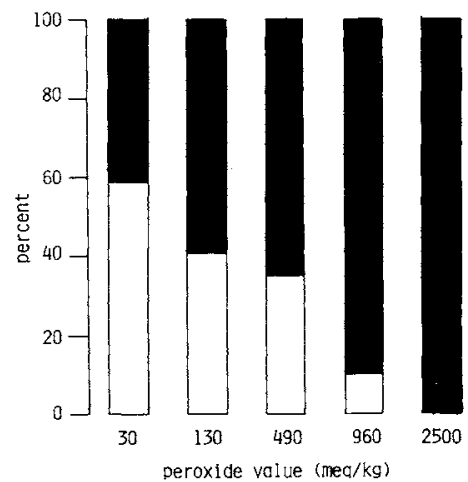

FIG. 4. The Proportion of Unsaturated Fatty Acyl Group of Olive Oil to the Formation of Hydroperoxy Fatty Acid Components.

- $, 18: 1 ; \square, 18: 2$.

The proportion of each fatty acyl group was calculated as follows:

$18: 2=(13-\mathrm{OH})+1.0 \times(13-\mathrm{OH})$

$18: 1=100-(18: 2)$

$1.0 \times(13-\mathrm{OH}): 9-\mathrm{OH}$ from $18: 2$.

scarcely seems to be involved in this experiment, because oxidation was carried out in the dark. However, it is likely that trace amounts of hydroperoxides accumulated by photosensitized oxidation during preservation prior to the autoxidation experiment. The proportion of the 12-isomer in the isomeric composition of soybean oil TG apparently is lower than that of soybean oil, as shown in Table II. The hydroperoxides and photosensitizer were removed during the preparation of TG. Thus, unsaturated TG including soybean oil TG did not give an unusual distribution of hydroperoxide isomers after autoxidation.

Table III shows the isomeric compositions 
of hydroperoxy fatty acid components obtained from autoxidized olive oil. From the results of Table I, the 8- and 10-isomers come from $18: 1$; the 9-isomer, from $18: 1$ and $18: 2$; the 13-isomer, from $18: 2$. Figure 4 shows the proportion of each unsaturated fatty acyl group of olive oil to the formation of isomeric hydroperoxides. The hydroperoxides coming from 18:2 decreased, and those coming from $18: 1$ increased with the oxidation level. At a higher level of oxidation, the isomeric distributions of $18: 1$ and $18: 2$, calculated from the scheme in the legend of Fig. 3, were in agreement with those of TO and TL, shown in Table I. However, at a lower level of oxidation (peroxide value $=30 \mathrm{meq} / \mathrm{kg}$ ), the 9 - and 10 isomers coming from 18:1 were significantly higher than those expected from the result of TO. According to the calculation in Fig. 3, $18: 1$ would yield the 8 -isomer $(3 \%)$, the 9 isomer $(14 \%)$, the 10 -isomer $(15 \%)$, and the 11 -isomer $(6 \%)$ in total hydroperoxides. This unusual distribution suggested that photosensitized oxidation participated in the formation of hydroperoxides, because the high proportions of the 9- and 10-isomers are characteristics for the photosensitized oxidation of $18: 1 .^{13)}$ Thus, it seems likely that isomeric hydroperoxides accumulated by photosensitized oxidation prior to the autoxidation experiments and affected the isomeric distribution of oxidized olive oil at a lower oxidation level.

The analysis of hydroperoxy fatty acid components of oxidized vegetable oils is useful not only in the determination of the proportion of each fatty acyl group to the formation of hydroperoxides, but also in the distinction between the oxidation mechanisms of free radical oxidation and photosensitized oxidation. The results of vegetable oil autoxidation in this work demonstrate that free radical oxidation proceeds in the production of the unsaturated fatty acyl group of TG in the isomeric hydroperoxides. Moreover, in the preceding paper $^{13)}$ we clarified that photosensitized oxidation participates in the for- mation of hydroperoxides during photooxidation of vegetable oil. Thus, it is apparent that trace amounts of hydroperoxides accumulated by the occurrence of photosensitized oxidation during the preservation of oils and that homolytic decomposition of hydroperoxides initiates the autocatalytic oxidation. The isomeric compositions of hydroperoxides in oxidized vegetable oils are considered to be related to the quality of the rancid odor, because most of volatile carbonyl compounds are formed from the isomeric hydroperoxides. In the next paper, HPLC are applied to the analysis of hydroperoxides of vegetable oils.

\section{REFERENCES}

1) E. N. Frankel, "Lipids and Their Oxidation" ed. by H. W. Schultz et al., AVI Publishing Co. Inc., 1962, p. 51 .

2) H. W.-S. Chan, Chem. Ind., 20, 692 (1977).

3) H. W.-S. Chan and G. Levett, Lipids, 12, 99 (1977).

4) H. W.-S. Chan and G. Levett, Lipids, 12, 837 (1977).

5) J. Terao and S. Matsushita, Agric. Biol. Chem., 39, 2027 (1975).

6) E. N. Frankel, W. E. Neff, W. K. Rohwedder, B. P. S. Khambay, R. F. Garwood and B. C. L. Weeden, Lipids, 12, 901 (1977).

7) E. N. Frankel, W. E. Neff, W. K. Rohwedder, B. P. S. Khambay, R. F. Garwood and B. C. L. Weeden, Lipids, 12, 908 (1977).

8) E. N. Frankel, W. E. Neff, W. K. Rohwedder, B. P. S. Khambey, R. F. Garwood and B. C. L. Weeden, Lipids, 12, 1055 (1977).

9) E. N. Frankel and W. E. Neff, Lipids, 14, 39 (1979).

10) K. Sohce, S. Izutani and S. Matsushita, Agric. Biol. Chem., 37, 1977 (1973).

11) K. Sohde, T. Ogawa and S. Matsushita, Yukagaku, 23, 228 (1974).

12) J. Terao and S. Matsushita, Agric. Biol. Chem., 42, 667 (1978).

13) J. Terao and S. Matsushita, Agric. Biol. Chem., 45, 601 (1981).

14) T. Asakawa and S. Matsushita, Lipids, 16, 965 (1981).

15) F. D. Gunstone and T. P. Hilditch, J. Chem. Soc., 1945, 836.

16) R. Yamauchi and S. Matsushita, Nippon Nôgeikagaku Kaishi, 52, 51 (1978).

17) L. R. Dugan, B. W. Beadle and A. S. Henick, J. Am. Oil Chem. Soc., 26, 681 (1949).

18) E. N. Frankel, W. E. Neff and T. R. Besslers, Lipids, 14, 961 (1979). 